\title{
RESEARCH
}

\section{Parental obesity programs pancreatic cancer development in offspring}

\author{
Raquel Santana da Cruz, Johan Clarke, Ana Cristina P Curi, Aseel Al-Yawar, Lu Jin, Ali Baird, M Idalia Cruz, \\ Bhaskar Kallakury and Sonia de Assis
}

Department of Oncology, Lombardi Comprehensive Cancer Center, Georgetown University, Washington, District of Columbia, USA

Correspondence should be addressed to S de Assis: deassiss@georgetown.edu

\begin{abstract}
Epidemiological studies suggest that timing of obesity onset - and underlying metabolic dysfunction - is important in determining pancreatic cancer rates: early and young adult abdominal overweight/obesity is more strongly associated with this cancer than obesity that develops later in life. Parental obesity and overweight are associated with metabolic dysfunction and obesity in their children. Here, we evaluated the impact of parental overweight on offspring's susceptibility of pancreatic cancer using the $P 48 \mathrm{Cre} /+/ \mathrm{Kras}_{\mathrm{G}} \mathrm{LD} /+$ mouse model. Male mice were fed an obesity-inducing diet (OID) before conception and mated with females raised on a control diet (CO) to generate the offspring. In a separate experiment, pregnant dams were fed CO or OID throughout gestation. The resulting OID offspring from the maternal (OID-m) or paternal lineage (OID-p) were used to study body weight, metabolic parameters and pancreatic cancer development and for molecular analysis. Parental obesity increased offspring's body weight at birth, weaning and in adulthood compared to CO, with gender- and genotype-specific differences. OID-p and OID-m offspring showed metabolic disorder and accelerated development of high-grade PanIN/PDAC. OID offspring also had higher rates of acinar-to-ductal reprogramming assessed by CPA1+/SOX9+-positive pancreatic cells. Levels of Tenascin C (TNC), an ECM glycoprotein shown to suppress apoptosis, were elevated in OID offspring, particularly females. In line with that, OID offspring displayed increased collagen content and decreased apoptosis in pancreatic lesions compared to CO. An ancestral history of obesity through either the paternal or maternal lineages increases offspring's susceptibility to pancreatic cancer development.
\end{abstract}

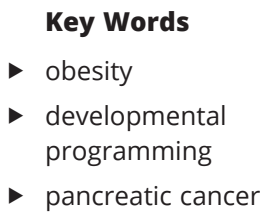

Endocrine-Related Cancer (2019) 26, 511-523

\section{Introduction}

Pancreatic ductal adenocarcinoma (PDAC) has an overall 5 -year survival rate of about 9\% (American Cancer Society 2018). This dismal prognosis is due to lack of early detection methods, effective therapies and our poor understanding of risk factors for this disease.

While presenting a ductal phenotype, PDAC has been experimentally shown to arise from the acinar compartment through a process of acinar-to-ductal reprogramming (Kopp et al. 2012). Kras mutations are present in early human PanIN lesions as well as invasive PDAC, and this activating mutation is generally accepted as the initiating event in PDAC progression (Kanda et al. 2013). However, PDAC development in mice carrying the KrasG120/+ mutation is slow (Hingorani et al. 2003), suggesting that other genetic or environmental factors are needed for tumor promotion (Dawson et al. 2013). 
There are few established risk factors for PDAC with family history, chronic pancreatitis, smoking, metabolic syndrome and obesity among them (Pandol et al. 2012). Epidemiologic studies suggest that obesity, overweight and underlying metabolic dysfunction account for up to $50 \%$ of all PDACs (Bracci 2012), and findings from animal studies support this association (Dawson et al. 2013, Chang et al. 2017). Population studies also suggest that timing of overweight/obesity onset is important in determining pancreatic cancer rates: childhood and young adult abdominal overweight/obesity is more strongly associated with this cancer than overweight/obesity that develops later in life (Li et al. 2009, Genkinger et al. 2015, Nogueira et al. 2017). However, the mechanisms behind this association are not well understood.

Parental environmental and life-style exposures have been shown to affect phenotypic characteristics in the next generation (Jirtle \& Skinner 2007). Most of the evidence comes from maternal exposures during pregnancy, given the intimate relationship between mother and the fetus (de Assis et al. 2006, Loche et al. 2018). However, recent studies demonstrate that pre-conception paternal factors can also modulate the risk of disease in their progeny $(\mathrm{Ng}$ et al. 2010, Rodgers et al. 2015). Children of obese parents are more likely to develop overweight, insulin resistance and diabetes in early life (Dorner \& Plagemann 1994, Linabery et al. 2013, Santos et al. 2018). These findings have been recapitulated in animal studies with overweight and metabolic dysfunction being observed in offspring for both maternal and paternal obesity (Jimenez-Chillaron et al. 2009, McPherson et al. 2015, Huypens et al. 2016).

Here, we evaluated the impact of diet-induced parental obesity in the susceptibility of pancreatic cancer in the next generation using the $\mathrm{P} 48^{\mathrm{Cre} /+} / \mathrm{Kras}^{\mathrm{G} 12 \mathrm{D} /+}$ (hereafter referred to as $\mathrm{KC}$ ) mouse model of pancreatic cancer. Our study shows that a history of obesity from either the paternal or maternal lineage is associated with accelerated development of highgrade PanIN and invasive PDAC in offspring carrying the Kras ${ }^{612 D /+}$ mutation. This phenotype was associated with increased rates of acinar-to-ductal reprogramming as well as increased desmoplasia and other alterations in the extracellular matrix (ECM) in a gender-specific manner.

\section{Experimental procedures}

\section{Animals and dietary exposures}

\section{Paternal exposures}

LSL-Kras ${ }^{G 12 D /+}$ or P48Cre/+ male mice were fed AIN93Gbased diets containing either $17.2 \%$ (Control, CO,
Envigo-Teklad \#TD.160018) or 57.1\% energy from fat (Lard-based, Obesity-Inducing-Diet, OID, Envigo-Teklad \#TD.160019) (Diet details in Supplementary Table 1, see section on supplementary data given at the end of this article) starting after weaning (3 weeks of age). Males' body weight was recorded weekly. At 10 weeks of age, OID-fed and control-fed male mice were mated to female mice (LSL-Kras ${ }^{\mathrm{G} 12 \mathrm{D} /+}$ or $\mathrm{P} 48^{\mathrm{Cre} /+}$ ) reared on the CO diet to generate the offspring. Males were kept in female cages for 3 days. Female mice were kept on the control diet during the breeding, for the extent of pregnancy and after giving birth. The weight and number of pups per litter was determined 2 days after birth. To avoid litter effect, pups were cross-fostered 2 days after dams gave birth. Pups from 2 to 3 dams were pooled and housed in a litter of 8-10 pups per nursing dam. All pups were weaned on postnatal day 21 and fed a standard chow diet throughout the experiment. Pups' body weight was recorded weekly.

\section{Maternal exposures}

LSL-Kras ${ }^{\mathrm{G}}{ }^{2 \mathrm{D} /+}$ or $\mathrm{P} 48^{\mathrm{Cr} / \mathrm{+}}$ females were fed the CO or OID starting 5 days before mating. OID-fed and CO-fed females were then housed together with male mice (LSL-Kras ${ }^{G 12 D /+}$ or $\mathrm{P} 48^{\mathrm{Cre} / \mathrm{t}}$ genotype) raised on the $\mathrm{CO}$ diet to mate for 3 days. Pregnancy onset was assessed by the presence of a vaginal plug. Maternal body weight was recorded weekly. OID-fed dams were switched to the CO diet, starting 2 days after delivery. Pups were cross-fostered and weaned as described earlier.

The offspring of CO or OID mothers and fathers were used to study body weight, metabolic parameters, molecular analyses and pancreatic tumorigenesis as described in the following sections.

All animal procedures were approved by the Georgetown University Animal Care and Use Committee, and the experiments were performed following the National Institutes of Health guidelines for the proper and humane use of animals in biomedical research. Animals were killed if symptoms of advanced tumor development (palpable mass, jaundice, sudden weight loss) occurred.

Genotyping The genotype of each animal was determined using genomic DNA extracted from tail clips and PCR using a commercially available service (Transnetyx, Inc., Cordova, TN, USA).

Pancreas histology Pancreatic tissue sections were fixed in neutral buffered $10 \%$ formalin, paraffin-embedded, sectioned $(5 \mu \mathrm{m})$ and stained with hematoxylin and eosin https://erc bioscientifica com https://doi.org/10.1530/ERC-19-0016 c) 2019 Society for Endocrinology Published by Bioscientifica Ltd. Printed in Great Britain 
(H\&E). PanINs and invasive ductal adenocarcinomas were classified blindly by our pathologist (B K) using $\mathrm{H} \& \mathrm{E}$ sections. The presence of PDAC and the highest grade (1, 2 or 3) of PanIN per section was recorded. To determine the number of PanIN per section, 20 random areas in H\&Estained slides were photographed at $20 \times$ magnification. The total number of PanIN lesions (1, 2 and 3)/section were counted and the ratio of high (PanIN 3)/low-grade (PanIN 1+2) was determined.

Metabolic parameters Glucose tolerance test was performed after overnight fasting, while insulin tolerance test was performed $2 \mathrm{~h}$ after diet removal in mice at 2 or 6 months of age. Glucose $(2.5 \mathrm{~g} / \mathrm{kg}$ body weight) or insulin $(0.75 \mathrm{U} / \mathrm{kg}$ body weight) were administered intraperitoneally and tail blood samples were collected at $0,15,30$ and 60 min after injection.

Pancreatic tissue collection Pancreatic tissue from control and OID offspring were collected at 2 months of age and again at 6-7 months ( $\geq 6$ months, hereafter) of age and used for pancreatic histopathology, immunohistochemistry analyses, RNA and protein extraction.

RNA sequencing RNA extraction was performed with the miRNeasy Mini Kit (Qiagen), according to the manufacturer's protocol, and its concentration, quality and integrity were confirmed using NanoDrop ND-1000 (Thermo Scientific). The quality of the samples was assessed using a 2100 Bioanalyzer (Agilent Technologies) for RNA integrity number $(>6.0)$ and concentration (minimum $60 \mathrm{ng} / \mu \mathrm{L}$ ). The RNA-seq analysis ( $n=6 /$ group) was performed by GENEWIZ (South Plainfield, NJ, USA).

\section{RNA library construction}

The RNA samples were quantified using Qubit 2.0 Fluorometer (Life Technologies) and RNA integrity was checked with Agilent TapeStation (Agilent Technologies). RNA library preparation, sequencing reaction and initial bioinformatics analysis were conducted at GENEWIZ, LLC. RNA sequencing library preparation used the NEBNext Ultra RNA Library Prep Kit for Illumina by following manufacturer's recommendations (NEB, Ipswich, MA, USA). Briefly, mRNAs were first enriched with oligod(T) beads. Enriched mRNAs were fragmented for $15 \mathrm{~min}$ at $94^{\circ} \mathrm{C}$. First-strand and second-strand cDNA were subsequently synthesized. cDNA fragments were end repaired and adenylated at 3'ends, and universal adapter was ligated to cDNA fragments, followed by index addition and library enrichment with limited cycle PCR. The sequencing library was validated on the Agilent TapeStation (Agilent Technologies) and quantified using Qubit 2.0 Fluorometer (Invitrogen) as well as by quantitative PCR (Applied Biosystems). The sequencing libraries were clustered on two lanes of a flowcell. After clustering, the flowcell was loaded on the Illumina HiSeq instrument according to manufacturer's instructions. The samples were sequenced using a $2 \times 150$ paired-end (PE) configuration. Image analysis and base calling were conducted by the HiSeq Control Software (HCS). Raw sequence data (.bcl files) generated from Illumina HiSeq was converted into fastq files and de-multiplexed using Illumina's bcl2fastq 2.17 software. One mismatch was allowed for index sequence identification.

\section{Data analysis}

All raw data passed the FastQC quality check. We used Rsem (Li \& Dewey 2011) with GRCm38 mouse reference sequence $(10 \mathrm{~mm})$ to assess gene expression level. We compared $\mathrm{KC}$ gene expression profile of control group to OID group. We also performed this analysis by gender (OID females vs control females; OID males vs control males). For all sets of comparisons, we performed differential expression analysis using limma package (Ritchie et al. 2015) in $\mathrm{R}$ and applied $\mathrm{fdr}<0.1$ as cut off point. A heatmap was generated for each set of filtered genes, which was then uploaded to IPA (Qiagen Inc., https:// www.qiagenbioinformatics.com/products/ingenuitypathway-analysis) for network and functional analysis.

\section{Validation of RNA-seq data by quantitative real-time polymerase chain reaction}

Differentially expressed genes identified (Lama1, Tff3 and Tnc) in our RNA-seq were validated through q-PCR. Total RNA from KC offspring pancreatic tissue was extracted ( $n=9-13$ /group) using the RNeasy Lipid Tissue Mini Kit per the manufacturer's protocol. The concentration, purity and quality of RNA samples were assessed as described earlier. Two micrograms of RNA per sample were used for the synthesis of cDNA using the High-Capacity cDNA Reverse Transcription Kit (Applied Biosystem) according to manufacturer's instructions. The expression levels of target genes were evaluated using a Step One Plus Real-Time PCR system (Applied Biosystems). The cDNA $(2 \mu \mathrm{g})$ was mixed with $5 \mu \mathrm{L}$ TaqMan Fast Advanced Master Mix (Applied Biosystem), 0.5 $\mu \mathrm{L}$ TaqMan Assay and $3.5 \mu \mathrm{L}$ 
ultrapure water (UltraPure DNase/RNase-Free Distilled Water) in 96-well plates. The amplification reactions were performed with a thermal cycling method, consisting of two initial cycles of $2 \mathrm{~min}$ at $50^{\circ} \mathrm{C}$ and $20 \mathrm{~s}$ at $95^{\circ} \mathrm{C}$, 40 cycles of $1 \mathrm{~s}$ at $95^{\circ} \mathrm{C}$ and $20 \mathrm{~s}$ at $60^{\circ} \mathrm{C}$. Expression of target genes were normalized to the housekeeping gene glyceraldehyde 3-phosphate dehydrogenase (Gapdh). The variation in the expression of target genes among experimental groups was analyzed using delta Ct.

\section{Immunohistochemistry and immunofluorescence staining}

Briefly, tissues were fixed in $10 \%$ buffered formalin, embedded in paraffin and sectioned $(5 \mu \mathrm{m})$. Sections were deparaffinized with xylene and rehydrated through a graded alcohol series. For immunohistochemistry staining, the antigen retrieval was performed by immersing the tissue sections at $98^{\circ} \mathrm{C}$ for 40 minutes in 1× Diva Decloaker (Biocare, Pacheco, CA, USA). Tissue sections were treated with $3 \%$ hydrogen peroxide and $10 \%$ normal goat serum for $10 \mathrm{~min}$ and were incubated with the primary antibody (Supplementary Table 2), overnight at $4^{\circ} \mathrm{C}$. After several washes, sections were treated with the appropriate HRP-labeled polymer for $30 \mathrm{~min}$ and DAB chromogen (Dako) for $5 \mathrm{~min}$. Slides were counterstained with hematoxylin (Fisher, Harris Modified Hematoxylin), blued using 1\% ammonium hydroxide, dehydrated and mounted with Acrymount. The sections were photographed using an Olympus IX-71 Inverted Epifluorescence microscope at 20x magnification. Images were evaluated with ImageJ software (National Institutes of Health, Bethesda, MD, USA) to quantify total pancreatic area, as well as the percentage of positive cells for CPA1, SOX9 and laminin. The proliferation index (ki67 staining) was determined by immunoRatio, a plugin for the ImageJ software to quantify hematoxylin and DAB-stained tissue.

For immunofluorescence staining, antigen retrieval (HIER) was performed by immersing the tissue sections in Target Retrieval Solution, low pH (Dako) in the PT Link (Dako). Tissue sections were treated with 3\% hydrogen peroxide, avidin/biotin blocking and 10\% normal horse serum and were incubated with the primary antibodies (Supplementary Table 2), for $1 \mathrm{~h}$ at room temperature. After several washes, sections were exposed to horse anti-goat-HRP (Vector Labs) and horse anti-rabbit-biotin (Vector) secondary antibodies, TSA-488 and Cy3-SA (Thermo Fisher) tertiaries and mounted in Pro-Long Antifade with DAPI (Thermo Fisher). Fluorescent images were scanned using the Vectra3 Multi-Spectral Imaging Microscope with Vectra and Phenochart software (Perkin Elmer). The entire slide was scanned, and then ten regions of interest were selected at random throughout the tissue. To ensure unbiased selection, the CPA1 and SOX9 or TNC fluors were turned off with DAPI remaining on to denote the tissue. The scanned images were analyzed in inform software version 2.3. Cells within each region of interest were identified and were phenotyped independently as $\mathrm{CPA} 1+/-$ and $\mathrm{SOX} 9+/-$ or as $\mathrm{TNC}+/-$. The individual phenotypes were combined in Excel to identify cell phenotypes as CPA1+/SOX9+, CPA1+/SOX9-, CPA1-/SOX9+, CPA1-/SOX9- or as TNC- and TNC+. An average of 20,000 cells was counted per slide.

\section{Western blot}

Protein levels were assessed by Western blot in the pancreatic tissue and tumors obtained from OID or control offspring. Total protein was extracted from pancreatic tissues and tumors using RIPA buffer with Halt Protease Inhibitor Cocktail (Thermo Fisher). Ten micrograms of protein extracts were resolved on a $4-12 \%$ denaturing polyacrylamide gel (SDS-PAGE). Proteins were transferred using the iBlot 7-Minute Blotting System (Invitrogen) and blocked with 5\% nonfat dry milk for $1 \mathrm{~h}$ at room temperature. Membranes were incubated with the specific primary antibodies (for antibody specifications and dilutions, see Supplementary Table 2) at $4^{\circ} \mathrm{C}$ overnight. After several washes, the membranes were incubated with horseradish peroxidase (HRP)conjugated secondary antibody at room temperature for 1 hour. Membranes were developed using the Chemiluminescent HRP antibody detection reagent (Denville Scientific Inc., Metuchen, NJ, USA) and exposed to blot imaging systems (Amersham Imager 600, GE Healthcare Life Sciences). Optical density of the bands was quantified using Quantity-one software (Bio$\mathrm{Rad})$. To control for equal protein loading, expression of the proteins of interest was normalized to the $\beta$-tubulin signal or cyclophilin signal.

\section{Collagen staining}

To evaluate collagen deposition, $5 \mu \mathrm{m}$ thick paraffinembedded pancreatic sections were stained with picrosirius red staining. Briefly, after de-waxing and hydration, tumor sections were stained with picro-sirius red solution ( $0.5 \mathrm{~g}$ of picro-sirius red F3B (C.I. 35782) in $500 \mathrm{~mL}$ of 
saturated aqueous solution of picric acid) for $1 \mathrm{~h}$. Sections were washed in two changes of acidified water, dehydrated in three changes of $100 \%$ ethanol, cleared in xylene and, then, mounted in a resinous medium. The sections were photographed using an Olympus IX-71 Inverted Epifluorescence microscope at $40 \times$ magnification. Staining was quantified by converting the image to gray scale and then isolating the red-stained collagen using thresholding and subsequent measure of the thresholded area with ImageJ software.

\section{Statistical analyses}

All statistical analyses were done using GraphPad Prism7 software. The results are presented as mean \pm s.E. Comparisons between groups were conducted using two-tailed Student's $t$ test (or corresponding nonparametric test). When comparing two variables for multiple conditions, a two-way ANOVA was performed. Time-courses measurements such as body weight and glucose tolerance test were analyzed by repeatedmeasures ANOVA. For comparison of frequency measurements such as cell phenotyping (CPA1+, SOX9+, TNC+) and PanIN/PDAC development, a chisquare test was performed. A $P$ value of less than 0.05 was considered significant.

\section{Results}

\section{Parental phenotypes}

Male mice fed OID gained more weight and were significantly heavier before breeding compared to males fed the CO diet (Supplementary Fig. 1A and B, $P<0.05$, $P=0.02)$. The increase in body weight was accompanied by an increase in circulating leptin $(P<0.09)$, but not in insulin, levels (Supplementary Fig. 1C and D).

Pregnant dams fed OID during gestation also gained more weight than those on the CO diet (Supplementary Fig. $2 \mathrm{~A}$ and $\mathrm{B}, P<0.05)$. However, circulating leptin and insulin levels were not different between groups (Supplementary Fig. 2C and D).

\section{Offspring phenotypes}

While both female and male (WT and KC combined) offspring of OID-fed fathers had a trend toward higher weight at birth (Fig. $1 \mathrm{~A}$ and $\mathrm{B}, P=0.07$ ), only OID-p female offspring were heavier at weaning (Fig. $1 \mathrm{C}$ and D, $P<0.05$ ) compared to CO. No major differences in body weight were observed after weaning in OID-p offspring (Fig. 1E, F, G and $\mathrm{H}$ ). However, we detected a non-significant increase in body weight in adult OID-p females from the KC genotype (Fig. 1F).
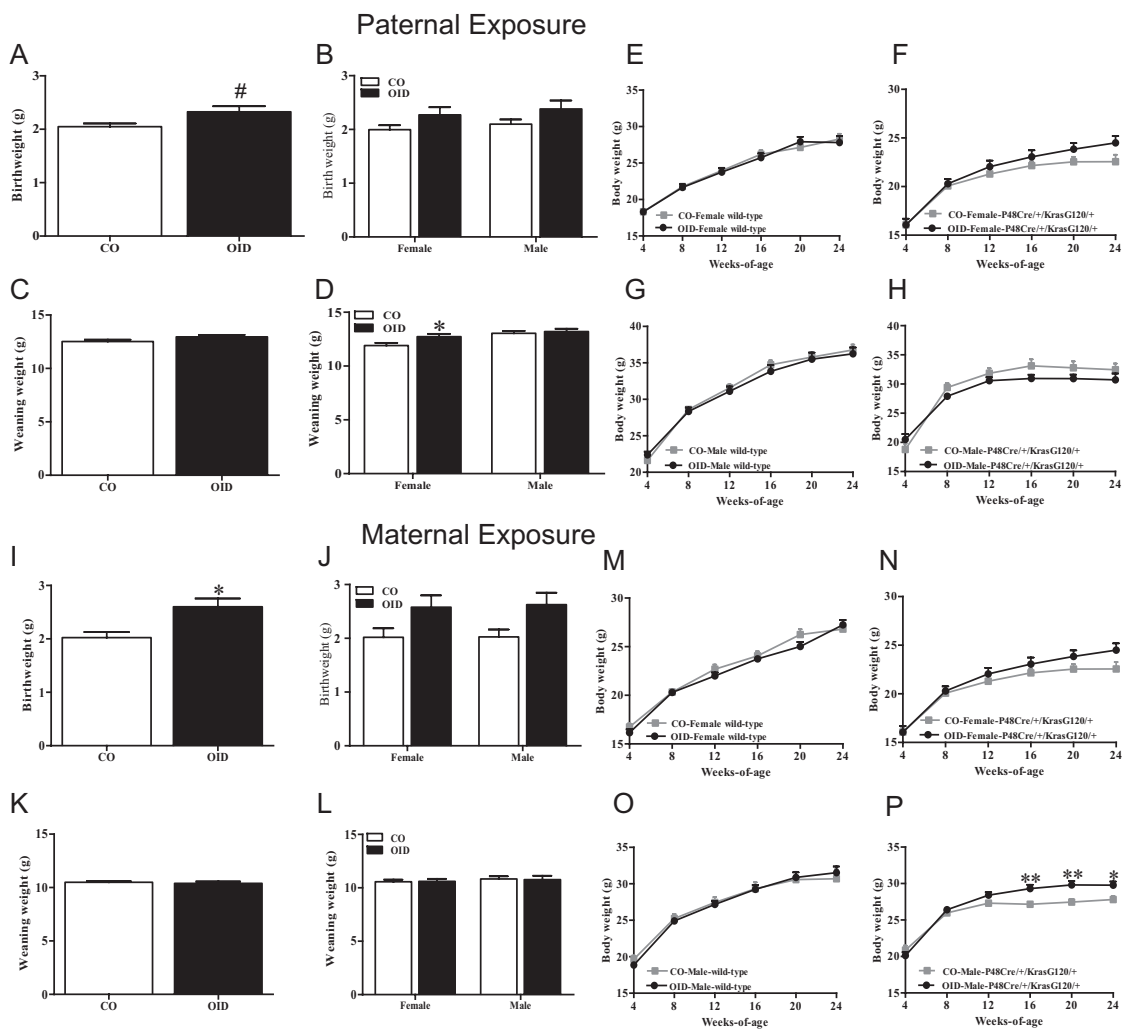

\section{Figure 1}

Body weight parameters in CO and OID offspring. ( $A, B, C, D, E, F, G$ and $H$ ) Birth weight ( $A$ and $B$ ), weaning weight ( $C$ and $D$ ) and longitudinal weight gain ( $E, F, G$ and $H$ ) in offspring from fathers fed Control (CO, $n=58$ ) or an obesity-inducing (OID, $n=52)$ diets; (I, J, K, L, M, N, O and P) Birth weight (I and J), weaning weight ( $K$ and $L$ ) and longitudinal weight gain (M, N, O and $\mathrm{P}$ ) in offspring from CO- $(n=44)$ and OID ( $n=34)$-fed mothers. The data are expressed as mean \pm S.E.M. Significant differences versus the $\mathrm{CO}$ group were determined by Student's $t$ test, two-way ANOVA or two-way repeated-measures ANOVA; ** $P<0.01, * P<0.05$ and ${ }^{\#} P=0.07$. 
Both female and male (WT and KC combined) offspring of OID-fed mothers had higher weight at birth compared to CO (Fig. 1I and J, $P=0.01$ ), but not at weaning (Fig. 1K and L). After puberty (Fig. 1M, N, O and $\mathrm{P})$, we detected an increase in body weight in both the $\mathrm{KC}$ females and males from the OID-m group (Fig. $1 \mathrm{~N}$ and $\mathrm{P})$, with statistically significant differences found only in OID-m males compared to CO (Fig. 1P, $P<0.01$ and $P<0.05)$

OID-p offspring showed impaired glucose tolerance at 2 (Fig. 2A, B and C, $P=0.02$ ) and 6 (Fig. 2D, E and F, $P=0.04$ ) months of age, particularly in males. OID-m offspring also showed impaired glucose tolerance at 2 (Fig. 2G, H and I, $P<0.01$ ) and at 6 (Fig. 2J, $\mathrm{K}$ and $\mathrm{L}, P<0.01$ ) months of age. Insulin intolerance was also detected in OID offspring at 6 months of age (Supplementary Fig. 3A, B and C, $P=0.01$ ). However, no differences in circulating insulin or leptin levels were detected between the groups (Supplementary Fig. 3D, E, F, G, H and I).

\section{Rates of PanIN and PDAC development in OID offspring}

All KC mice in either the CO or OID offspring groups developed PanIN and PDAC as previously described (Hingorani et al. 2003). The tumorigenesis process, however, was accelerated in the OID offspring from both the paternal or maternal OID lineages (Fig. 3A, B, C, D, E, F, G, H, I, J, K, L, M, N, O, P, Q, R, S, T, U, V, W and X).

At 2 months of age, the OID-p offspring had increased ratio of high/low-grade PanIN compared to $\mathrm{CO}$ (Fig. 3E and $\mathrm{F}, P=0.02)$. The OID-p offspring also had a nonsignificant increase in numbers of invasive PDAC (Fig. 3G and $H, P=0.2$ ) at this time point, with 50\% of OID-p mice
A
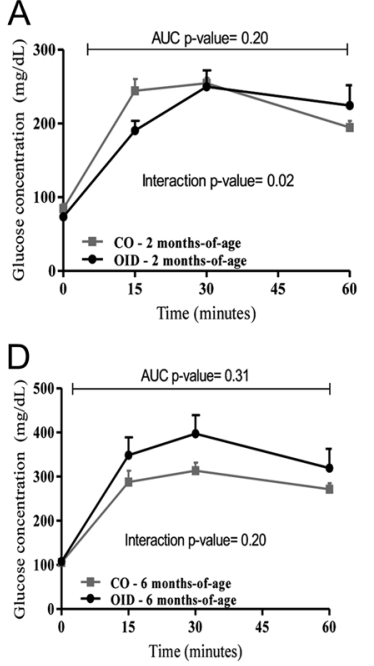

G

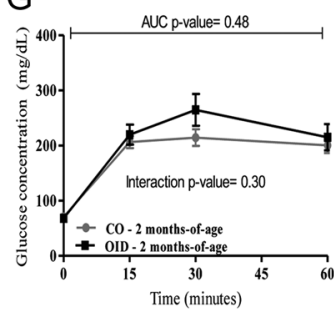

$J$

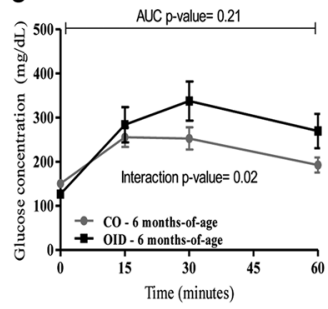

Paternal Exposure

B
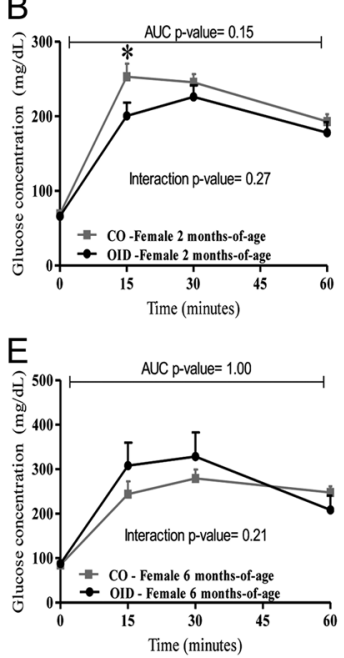

Maternal Exposure

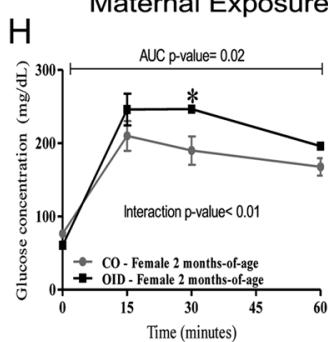

K

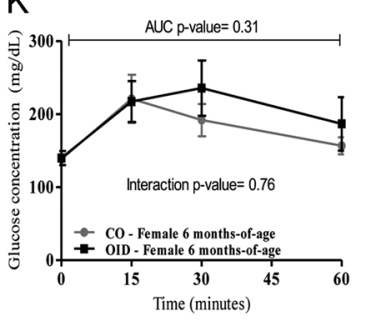

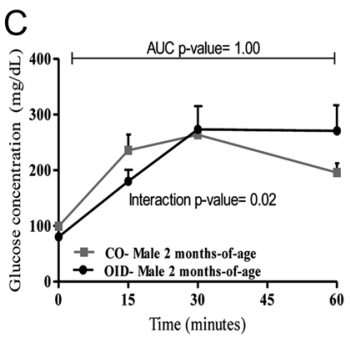
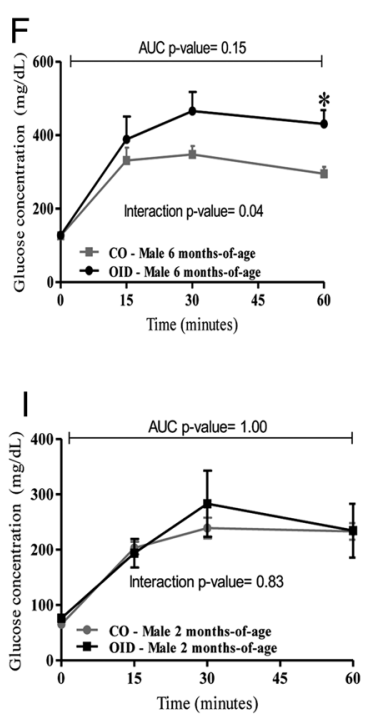

$\mathrm{L}$

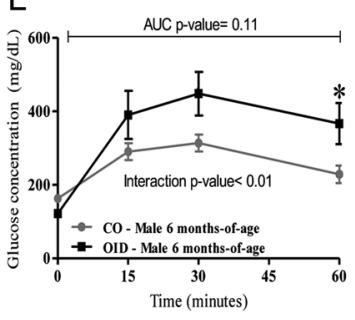

Figure 2

Metabolic function in $\mathrm{CO}$ and OID offspring. (A, B, C, D, E and F) Glucose tolerance test (GTT) in offspring from CO- and OID-fed fathers at 2 months ( $A$, all offspring; $B$, females; $C$, males) and at 6 months ( $D$, all offspring; $E$, females; $F$, males) of age. ( $G, H, I, J, K$ and $L) ~ G T T$ in offspring from CO- and OID-fed mothers at 2 months ( $G$, all offspring; $\mathrm{H}$, females; I, males) and 6 months (U, all offspring; $K$, females; $L$, males) of age from $\mathrm{CO}$ and OID-fed mothers ( $n=5 /$ gender/age/group). The data are expressed as mean \pm S.E.M. Significant differences versus the $\mathrm{CO}$ group were determined by two-way repeated-measures ANOVA; $* P<0.05$. 
developing PDAC, while none were observed in the $\mathrm{CO}$ group. At 6 months of age and older, we continued to observe accelerated development of pancreatic lesions, with increased ratio of high/low-grade PanIN in the OID-p offspring compared to CO (Fig. 3I and J, $P=0.01$ ). Slightly higher number of invasive PDACs in the OID-p group was also observed in this age cohort; however, results did not reach statistical significance (Fig. $3 \mathrm{~K}$ and L).

PanIN and PDAC development in OID-m offspring (Fig. 3M, N, O, P, Q, R, S, T, U, V, W and X) largely mirrored what was observed for the OID-p offspring. At 2 months of age, the OID-m offspring had increased ratio of high/lowgrade PanIN (Fig. 3Q and $\mathrm{R}, P=0.05$ ), particularly in females (Fig. 3R, $P<0.05$ ). A non-significant increase in numbers of invasive PDAC in OID males compared to $\mathrm{CO}$ was also found (Fig. 3S and T). At 6 months of age and older, we continued to observe accelerated development of lesions, with increased ratio of high/low-grade PanIN in OID-m offspring compared to $\mathrm{CO}$ (Fig. $3 \mathrm{U}, \mathrm{V}$ and $\mathrm{W}, P=0.02$ ), mainly in females $(P<0.01)$. Although not statistically significant, higher number of invasive PDACs was also observed in the OID-m offspring at this age cohort, with all PDAC detected in males (Fig. $3 \mathrm{~V}, \mathrm{~W}$ and $\mathrm{X}$ ).

Given the overlap in PanIN/PDAC phenotype between offspring produced from maternal and paternal OID exposures, all subsequent analyses were combined, with the OID-m and OID-p offspring collectively referred to as OID from this point on.
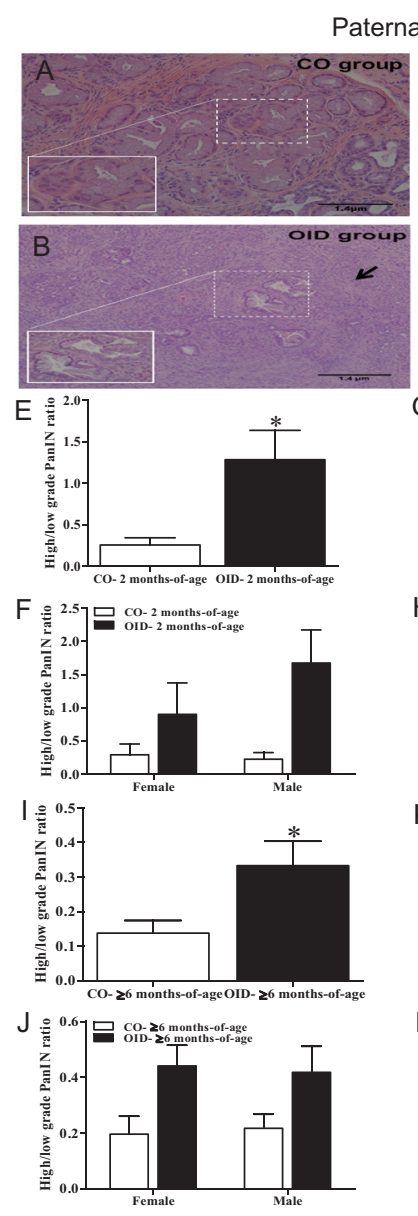

aternal Exposure
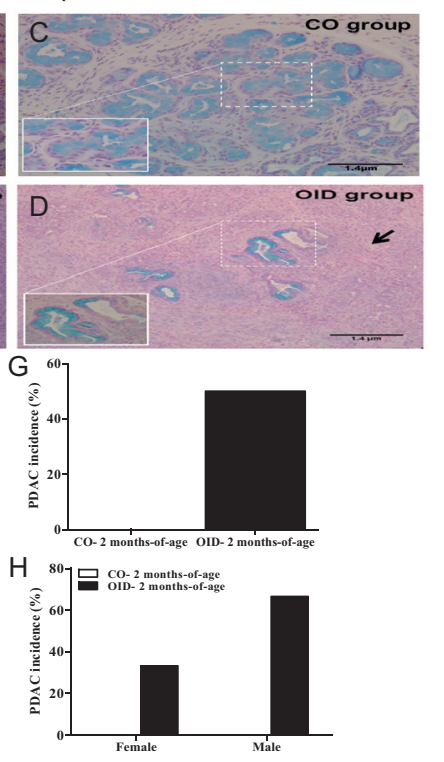

$\mathrm{K}$
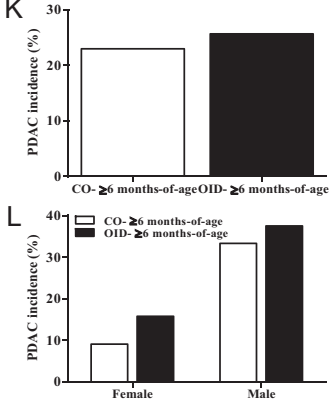
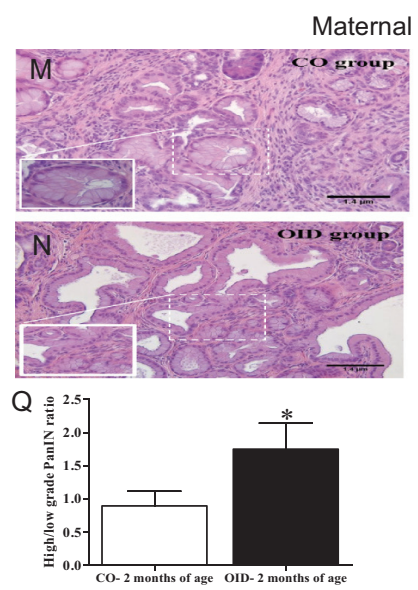

$\mathrm{R}$
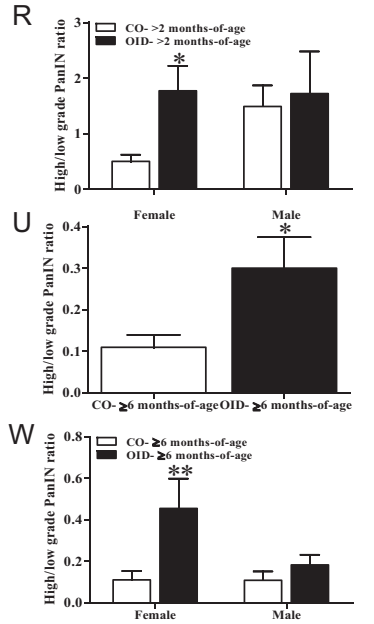
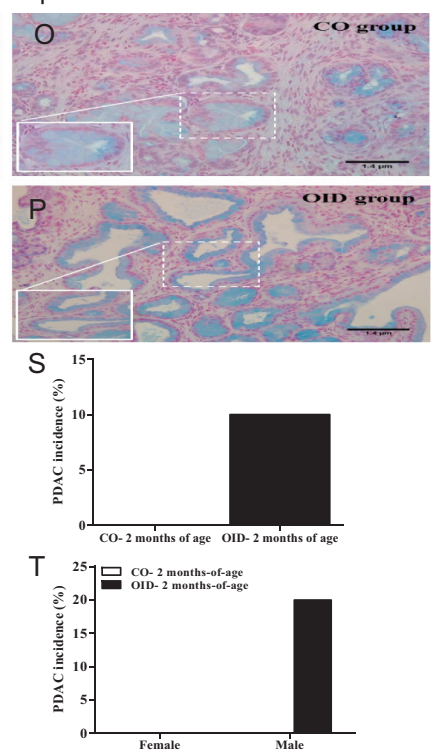

$\mathrm{V}$
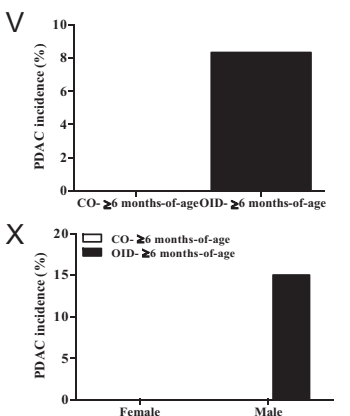

Figure 3

Pancreatic ductal adenocarcinoma development (PDAC) in CO and OID offspring. (A, B, C and D) Representative pancreas histopathology sections from offspring of CO- and OID-fed fathers at 2 months of age ( $A$ and $B, H \& E$ staining; $C$ and $D$, Alcian blue staining); ( $E, F, G, H, I, J, K$ and $L$ ) Ratio of high/ low-grade PanIN and invasive PDAC incidence in offspring of CO- and OID-fed fathers at $2(E, F, G$ and $H)$ and $\geq 6$ months (I, J, K and L) of age. (M, N, O and P) Representative pancreas histopathology sections from offspring of CO- and OID-fed mothers at 2 months of age (M and $N$, H\&E staining; O and $P$, Alcian blue staining); (Q, R, S, T, U, V, W and X) Ratio of high/low-grade PanIN and invasive PDAC incidence in offspring of CO- and OID-fed mothers at 2 $(\mathrm{Q}, \mathrm{R}, \mathrm{S}$ and $\mathrm{T})$ and $\geq 6$ months $(\mathrm{U}, \mathrm{V}, \mathrm{W}$ and $\mathrm{X})$ of age. The data are expressed as mean \pm S.E.M. Significant differences versus the CO group were determined by two-way ANOVA, Student's $t$ test or chi-square test; $* * P<0.01$ and $* P<0.05$. Inset, PanIN lesions; arrows, invasive PDAC lesion. Scale bars: $1.4 \mu \mathrm{m}$. A full colour version of this figure is available at https://doi.org/10.1530/ERC-19-0016. 


\section{Rates of proliferation and apoptosis in OID offspring PanIN and PDAC}

The KRAS signaling pathway (MAPK activation) (Supplementary Fig. 4A and B) is increased in OID offspring pancreas compared to CO. Thus, we evaluated pancreatic cell proliferation and apoptosis rates in KC OID offspring (Fig. 4A, B, C, D, E, F, G, H, I, J, K, L and M). While we did not find differences in cell proliferation levels (ki67 staining) between the groups (Fig. 4E, F and G), we detected a reduction in apoptosis levels (Fig. $4 \mathrm{H}$, I and J) in OID males at $2(P<0.001)$ and in both OID genders at 6 months of age $(P<0.01)$ compared to CO. We also found a concomitant increase in the pancreatic proliferation-to-apoptosis (Fig. $4 \mathrm{~K}$, $\mathrm{L}$ and $\mathrm{M}$ ) ratio for the OID offspring at 2 and 6 months of age, but results did not reach statistical significance.

\section{Increased rates of acinar-to-ductal reprogramming in OID offspring}

It has been shown that acinar-to-ductal reprogramming is the main mechanism by which pancreatic ductal carcinoma arises (Kopp et al. 2012). Given the higher rates of PanIN/PDAC progression in OID offspring, we wondered whether pancreatic acinar-to-ductal reprogramming was also accelerated. Using co-staining for CPA1 (acinar cell marker) and SOX9 (inducer of ductal cell identity), we observed significantly higher rates of acinar-to-ductal reprogramming (CPA1+, SOX9+ cells) in pancreata of $\mathrm{KC}$ OID offspring compared to $\mathrm{CO}$ both at 2 and 6 months of age (Fig. 5A, B, C, D, E, F, G and H and Supplementary Fig. $5, P<0.001, P<0.001)$.

\section{ECM alterations in OID offspring}

Given the accelerated PDAC progression and acinar-toductal reprogramming in KC OID mice, we investigated whether there were other differences in pancreatic gene expression patterns between the groups (6 months of age) that could explain our findings. Surprisingly, we found that a comparison between the $\mathrm{CO}$ and OID offspring yielded little differences (three annotated genes, data not shown) when male and females in each group were analyzed together. When the analysis was
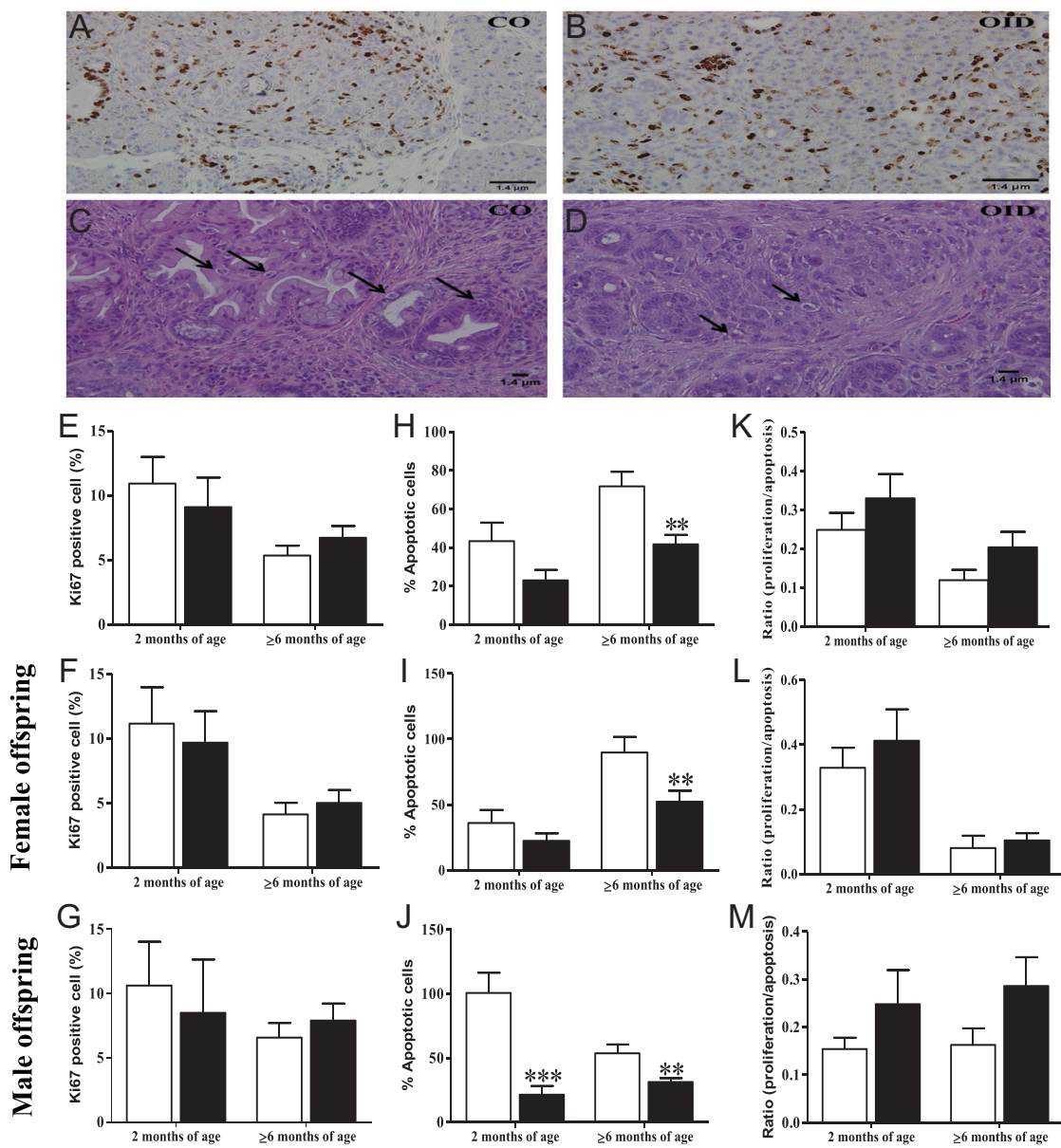

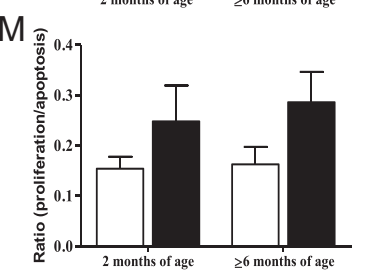

Figure 4

Cell proliferation and apoptosis in pancreatic tissue of CO and OID offspring. (A, B, C and D) Representative proliferation (Ki67, A and B) and apoptosis ( $\mathrm{H} \& \mathrm{E}$, morphological assessment, $\mathrm{C}$ and D) staining in pancreas sections from $\mathrm{KC} \mathrm{CO}$ and OID offspring at 2 months of age. ( $E, F$ and $G$ ) Quantification (\%) of proliferating cells in KC offspring's pancreas ( $E$, all offspring; $F$, females; $G$, males) at 2 ( $n=6 /$ group) and 6 months ( $n=10$ / group) of age. ( $\mathrm{H}, \mathrm{I}$ and J) Quantification (\%) of apoptotic cells in KC CO and OID offspring $(\mathrm{H}$, all offspring; I, females; J, males) at 2 ( $n=9-13$ /group) and 6 months ( $n=14-22$ /group) of age. (K, L and M) Proliferation/apoptosis ratio ( $K$, all offspring; $L$, females; $M$, males) in KC CO and OID offspring at 2 and 6 months of age. Randomly selected areas (20x magnification) were analyzed using immunoRatio software. The data are expressed mean \pm S.E.M. Significant differences versus the CO group were determined by two-way ANOVA; $* \star P<0.01$ and $* * * P<0.001$. Arrows, apoptotic cells. Scale bars: $1.4 \mu \mathrm{m}$. A full colour version of this figure is available at https://doi.org/10.1530/ ERC-19-0016. 

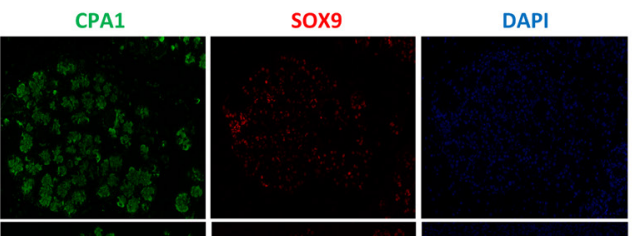

E

$\mathrm{F}$
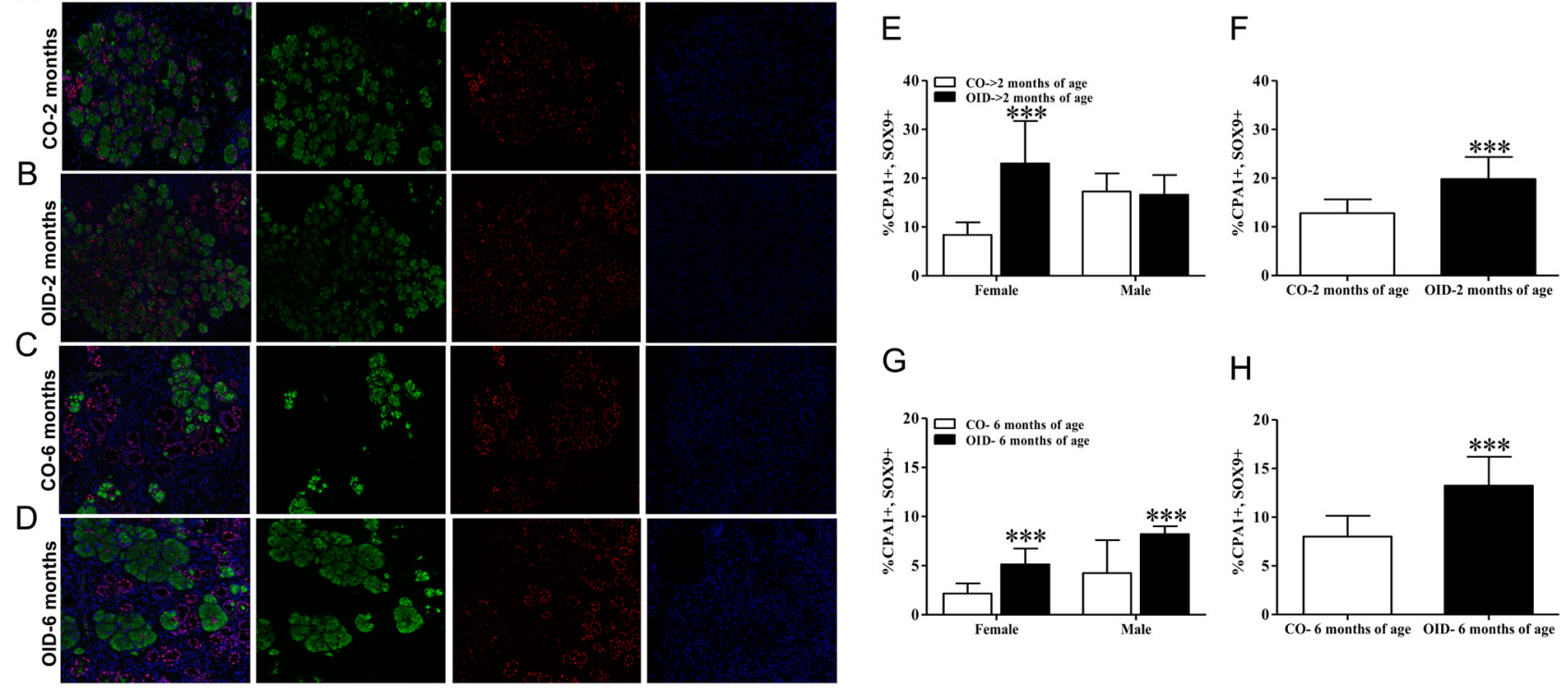

G

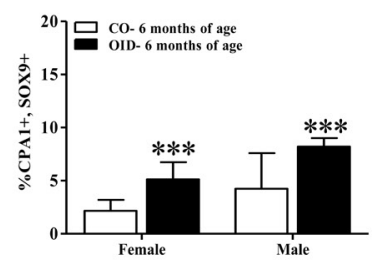

$\mathrm{H}$

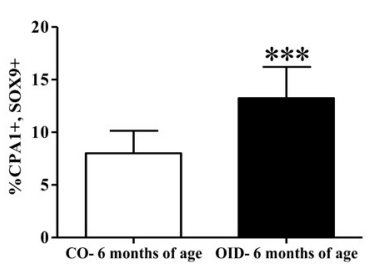

Figure 5

Assessment of acinar-to-ductal reprogramming (double immunofluorescence labeling of CPA1+/SOX9+) in pancreatic tissue of CO and OID offspring. (A, $B, C$ and D) Representative CPA1+ and SOX9+ staining in pancreas sections from offspring of KC CO and OID at 2 (A and B) and 6 months (C and D) of age. $(\mathrm{E}, \mathrm{F}, \mathrm{G}$ and H) Quantification (\%) of CPA1+/SOX9+ cells in KC offspring's pancreas ( $n=6 /$ age/group) at 2 ( $\mathrm{E}$, by gender; F, all offspring) and 6 (G, by gender; $\mathrm{H}$, all offspring) months of age. An average of 20,000 cells/section (20x magnification) were analyzed using the Vectra3 Multi-Spectral Imaging System.

The data are expressed mean \pm S.E.M. Significant differences versus the CO group were determined by chi-square test; $* \star \star P<0.001$.

performed by gender, however, several genes showed differential expression in KC OID offspring compared to $\mathrm{CO}$, particularly in females. Results are presented in heat-maps in Supplementary Fig. 6. A pathway analyses (Supplementary Fig. 7) revealed 'organismal injury' and 'connective tissue function/disorders' as some of the main bio-functions altered in OID offspring. Genes associated with those functions and upregulated in OID offspring included Tnc, Lama1 and Tff3.

An increase in Tnc (Tenascin C) expression was observed only in OID females in the RNA-seq analysis. Validation by q-PCR confirmed those results (Fig. 6A and $\mathrm{B}, P=0.02)$. Also, in agreement with the RNA-seq results, q-PCR validation showed that Lama1 was significantly upregulated in OID female pancreas (Fig. 6C and D, $P<0.001)$ only. Validation of Tff3 expression by q-PCR, on the other hand, revealed a non-significant increase in both male and female OID offspring's pancreas compared to CO (Fig. 6E and F). Next, we measured the protein levels of TNC using immunofluorescence and, in line with the RNA-seq results, found increased TNC expression $(P>0.001)$ in OID female, but not male, offspring pancreatic tissues at 6 months of age compared to $\mathrm{CO}$ (Fig. 6G and H). We also quantified protein of levels of laminin (LAMA1) using immunohistochemistry, and while we detected an increase expression in OID offspring pancreases, the results were not statistically significant (Fig. 6I and J).

PDAC is characterized by desmoplastic reaction (Whatcott et al. 2015). Given that in pancreatic tissue of OID offspring some differentially expressed genes were associated with connective tissue function/ disorder, we investigated whether there were differences in desmoplasia between groups. Using picro-sirius red staining (collagen marker), we observed that pancreatic tissues of KC OID offspring displayed increased collagen content at 6 months of age (Fig. $6 \mathrm{~K}$ and L, $P<0.01$ ).

\section{Discussion}

Using the KC mouse model of pancreatic cancer, we demonstrated that parental obesity in pre-conception (paternal) or in pregnancy (maternal) is linked to accelerated PanIN/PDAC development in the next generation. This increase in PDAC development was associated with higher rates of acinar-to-ductal reprogramming, reduced apoptosis rates and ECMrelated changes, including higher desmoplasia and TNC expression (in females) in pancreatic tissues.

There is strong evidence that parental obesity is linked to metabolic dysfunction and overweight in the 
A

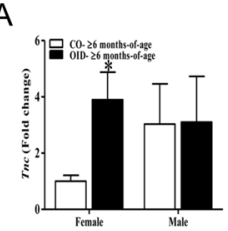

C
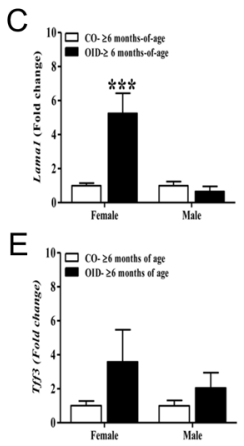

M

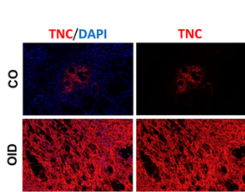

B
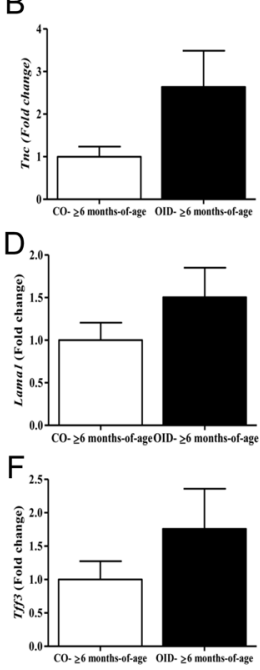

N

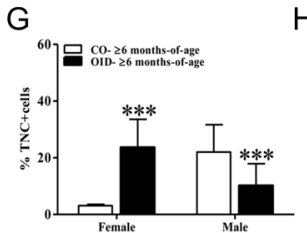

$\mathrm{H}$
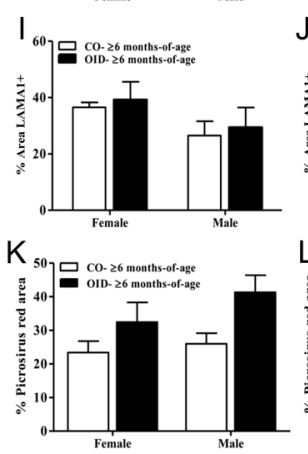

$\mathrm{O}$
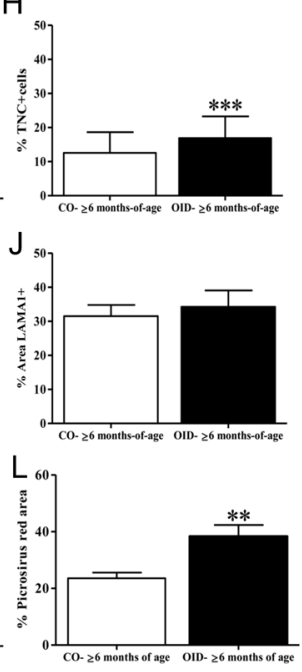
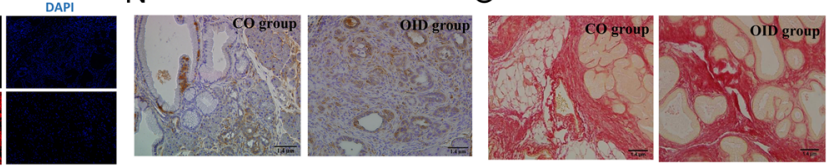

\section{Figure 6}

Assessment of ECM-related alterations in pancreatic tissue of $C O$ and OID offspring. (A, B, C, $D, E$ and $F$ ) mRNA expression (fold change) of Tnc (A, by gender; B, all offspring), Lama1 (C, by gender; $D$, all offspring) and Tff3 ( $E$, by gender; $F$, all offspring) in pancreatic tissue from $\mathrm{KC} C \mathrm{CO}$ and OID offspring ( $n=5-8$ /group) by q-PCR. ( $\mathrm{G}$ and $\mathrm{H}$ ) Quantification (\%) of immunofluorescence labeling of TNC+ areas ( $\mathrm{G}$, by gender; $\mathrm{H}$, all offspring) in pancreatic tissue from $\mathrm{KC} \mathrm{CO}$ and OID offspring ( $n=6 /$ group). (I and J) Quantification (\%) of LAMA 1+ areas (I, by gender; J, all offspring) in pancreatic tissue from KC CO and OID offspring ( $n=6$ /group) by IHC. (K and L). Quantification (\%) of collagen staining (picro-sirius red) ( $K$, by gender; $L$, all offspring) in pancreatic tissue from KC CO and OID offspring ( $n=25-27 /$ group) (mean \pm S.E.M.). All assessments were performed at $\geq 6$ months of age. (M, N and O) Representative sections showing staining for TNC (M), LAMA1 (N) and collagen (O). TNC staining was quantified using the Vectra3 Multi-Spectral Imaging System. LAMA1 and collagen stainings were analyzed using image J software. The data are expressed mean \pm S.E.M. Significant differences versus the CO group were determined by two-way ANOVA, Student's $t$ test or chi-square test; $* \star \star P<0.001$, $\star \star P<0.01$ and $* P<0.05$. next generation in humans (Dorner \& Plagemann 1994, Linabery et al. 2013, Santos et al. 2018). These findings have been recapitulated in animal studies (JimenezChillaron et al. 2009, McPherson et al. 2015, Huypens et al. 2016). Our study supports those findings as we detected an increase in early life body weight as well as alterations in metabolic function for both OID-p and OID-m offspring.

In population studies, obesity/overweight and diabetes are consistently linked to PDAC (Bracci 2012, Eibl et al. 2018). Obesity and diabetes often co-exist, but can also independently increase the risk for PDAC (Jiao et al. 2010, Eibl et al. 2018). The timing of obesity has been shown to affect the association with PDAC: central obesity and higher BMI in childhood and young adulthood is associated with greater risk of pancreatic cancer and earlier disease onset compared to normal weight individuals or those who are normal weight or gain more weight later in life (Li et al. 2009, Genkinger et al. 2015, Nogueira et al. 2017). Our findings offer support to those population studies. Further, our results also suggest that metabolic dysfunction in early life accelerates PDAC development, particularly in males. However, the relationship between pancreatic cancer and diabetes is complex as diabetes can play both a causative role and also be a consequence of pancreatic cancer (Risch 2019). Given that the OID offspring with the WT genotype had metabolic alterations, it is likely that the metabolic dysfunction in our model is causal and not the end result of PDAC development. While those findings need to be further investigated in animal models, it will be important to determine whether in patients with familial pancreatic cancer, early life metabolic dysfunction by itself or underlying obesity would accelerate PDAC onset.

We have previously shown that ancestral obesity or nutritional patterns can increase other types of cancer in offspring (de Assis et al. 2012, Fontelles et al. 2016, da Cruz et al. 2018). This present study shows that parental obesity can predispose their children to PDAC as well. We also detected gender-specific effects in OID offspring. The metabolic dysfunction in OID offspring was more readily detectable in OID males compared to OID females. In line with that, most of the invasive PDACs were observed in OID males. However, OID females also had significantly higher rates of high-grade PanINs than controls females, suggesting that the oncogenesis process is accelerated within this group as well.

There are striking similarities in the phenotypic changes as well in PDAC development in both the offspring from overweight fathers and mothers, indicating that the triggering mechanisms behind those phenotypes (c) 2019 Society for Endocrinology Published by Bioscientifica Ltd. Printed in Great Britain 
are likely the same for either ancestral lineage. While we have not performed mechanistic studies, some reports suggest that a common alteration underlying paternal and maternal exposures' effects on offspring could be alterations in placenta development (Rosenfeld 2015, Watkins et al. 2017). Further, we and others have shown that paternal obesity and nutrition leads to changes in sperm small non-coding RNAs (Fontelles et al. 2016, da Cruz et al. 2018) in rodents and humans (Donkin et al. 2016). The small RNAs present in sperm have been shown to alter embryonic development after fertilization in animals (Sharma et al. 2016).

Interestingly, while both OID fathers and mothers gained more weight than controls, levels of leptin were higher in OID fathers only. There are two possible explanations for this finding. First, fathers were fed the OID for a longer period of time than mothers (7 weeks versus $\sim 4$ weeks). More likely, however, the reason why we did not observe increased leptin levels in OID mothers is that this adipokine increases with pregnancy (Domali \& Messinis 2002) and would have increased in control pregnant mothers as well, masking the effects of increased body weight.

Although having a ductal phenotype, PDAC has been experimentally shown to arise from the acinar compartment through a process of acinar-to-ductal reprogramming (Kopp et al. 2012). Using cell populationspecific tracing experiments, a recent study showed that this process depends on ectopic expression of the ductal gene Sox9 in acinar cells that, in the presence of mutation oncogenic Kras, progress to PanIN (Kopp et al. 2012). Our data show that OID offspring have greater numbers of $\mathrm{CPA} 1+/ \mathrm{SOX} 9+$ cells, indicating that acinar cells transition more quickly to a ductal-like state, in the presence of oncogenic Kras. Whether this is the case in young individuals that are overweight/obese or have metabolic dysfunction still needs to be investigated. Studies also have shown that SOX9 expression is activated by the MAPK pathway (Ling et al. 2011), which is in line with the increased activation of this pathway in OID offspring.

ECM abnormality is one of the hallmarks of cancer (Ritchie et al. 2015), and it has been demonstrated that obesity increases desmoplasia in pancreatic cancer, interfering with therapeutic efficacy (Incio et al. 2016). Further, Tenascin $\mathrm{C}$ has been shown to suppress apoptosis and rescue pancreatic cancer cells from gemcitabineinduced apoptosis (Shi et al. 2015). In line with that, our studies suggest that having a history of ancestral obesity leads to higher collagen deposition and other
ECM-related alterations and reduced apoptosis levels in pancreatic tissues.

In this study, OID offspring were fed a balanced diet throughout their lives and whether postnatal dietary intake and other lifestyle changes can further modulate PDAC development still needs to be evaluated. It will be important also to elucidate if the pancreatic tissue environment or systemic changes are driving the increased PDAC observed in OID offspring. Metabolic disorders such obesity and diabetes are characterized by low-grade inflammatory state with increased levels of adipokines and pro-inflammatory cytokines (Monteiro \& Azevedo 2010, Eibl et al. 2018), creating conditions for cancer development (van Kruijsdijk et al. 2009).

The control diet in our study has a high carbohydrate, moderate fat profile, considered a balanced dietary pattern (Odermatt 2011). This control diet is clearly more beneficial to both parents and offspring health compared to the OID. While more comprehensive studies in both in humans and animal models are needed, those dietary patterns may possibly be recommended to parents trying to conceive as means to reduced metabolic diseases and cancer in the next generation.

Our study is the first to describe that parental obesity in pre-conception (fathers) or in pregnancy (mothers) is associated with accelerated development of PDAC in the next generation. Our study gives support to epidemiologic findings suggesting that childhood and early life overweigh/obesity and metabolic dysfunction are more strongly associated with pancreatic cancer than obesity that develops later in life. Interventions to reduce adiposity and metabolic dysfunction in early life may be an effective way to prevent pancreatic cancer and likely other malignancies.

\section{Supplementary data}

This is linked to the online version of the paper at https://doi.org/10.1530/ ERC-19-0016.

\section{Declaration of interest}

The authors declare that there is no conflict of interest that could be perceived as prejudicing the impartiality of the research reported.

\section{Funding}

This research was supported by the National Institutes of Health (CA178309) and pilot funding from the National Center For Advancing Translational Sciences of the National Institutes of Health (UL1TR001409) to Sonia de Assis. This research was also supported by the National Institutes of Health Lombardi Comprehensive Cancer Center Support Grant (P30-CA51008). 


\section{Acknowledgments}

The authors thank the Lombardi Cancer Center Shared Resources (SR) for their assistance: Animal Model SR, Histopathology and Tissue SR, Microscopy and Imaging SR, Genomics and Epigenomics SR and PIRL.

\section{References}

American Cancer Society 2018 Cancer facts \& figures 2018. Atlanta, GA, USA: American Cancer Society. (available at: https://www.cancer.org/ content/dam/cancer-org/research/cancer-facts-and-statistics/annualcancer-facts-and-figures/2018/cancer-facts-and-figures-2018.pdf)

Bracci PM 2012 Obesity and pancreatic cancer: overview of epidemiologic evidence and biologic mechanisms. Molecular Carcinogenesis 51 53-63. (https://doi.org/10.1002/mc.20778)

Chang HH, Moro A, Takakura K, Su HY, Mo A, Nakanishi M, Waldron RT, French SW, Dawson DW, Hines OJ, et al. 2017 Incidence of pancreatic cancer is dramatically increased by a high fat, high calorie diet in KrasG12D mice. PLoS ONE 12 e0184455. (https://doi.org/10.1371/journal.pone.0184455)

da Cruz RS, Carney EJ, Clarke J, Cao H, Cruz MI, Benitez C, Jin L, Fu Y, Cheng ZL, Wang Y, et al. 2018 Paternal malnutrition programs breast cancer risk and tumor metabolism in offspring. Breast Cancer Research 20 99. (https://doi.org/10.1186/s13058-018-1034-7)

Dawson DW, Hertzer K, Moro A, Donald G, Chang HH, Go VL, Pandol SJ, Lugea A, Gukovskaya AS, Li G, et al. 2013 High-fat, highcalorie diet promotes early pancreatic neoplasia in the conditional KrasG12D mouse model. Cancer Prevention Research 6 1064-1073. (https://doi.org/10.1158/1940-6207.CAPR-13-0065)

de Assis S, Khan G \& Hilakivi-Clarke L 2006 High birth weight increases mammary tumorigenesis in rats. International Journal of Cancer 119 1537-1546. (https://doi.org/10.1002/ijc.21936)

de Assis S, Warri A, Cruz MI, Laja O, Tian Y, Zhang B, Wang Y, Huang TH \& Hilakivi-Clarke L 2012 High-fat or ethinyl-oestradiol intake during pregnancy increases mammary cancer risk in several generations of offspring. Nature Communications 3 1053. (https://doi. org/10.1038/ncomms2058)

Domali E \& Messinis IE 2002 Leptin in pregnancy. Journal of MaternalFetal and Neonatal Medicine 12 222-230. (https://doi.org/10.1080/ jmf.12.4.222.230)

Donkin I, Versteyhe S, Ingerslev L, Qian K, Mechta M, Nordkap L, Mortensen B, Appel E, Jørgensen N, Kristiansen V, et al. 2016 Obesity and bariatric surgery drive epigenetic variation of Spermatazoa in humans. Cell Metabolism 23 369-378. (https://doi.org/10.1016/j. cmet.2015.11.004)

Dorner G \& Plagemann A 1994 Perinatal hyperinsulinism as possible predisposing factor for diabetes mellitus, obesity and enhanced cardiovascular risk in later life. Hormone and Metabolic Research 26 213-221. (https://doi.org/10.1055/s-2007-1001668)

Eibl G, Cruz-Monserrate Z, Korc M, Petrov MS, Goodarzi MO, Fisher WE, Habtezion A, Lugea A, Pandol SJ, Hart PA, et al. 2018 Diabetes mellitus and obesity as risk factors for pancreatic cancer. Journal of the Academy of Nutrition and Dietetics 118 555-567. (https://doi. org/10.1016/j.jand.2017.07.005)

Fontelles CC, Carney E, Clarke J, Nguyen NM, Yin C, Jin L, Cruz MI, Ong TP, Hilakivi-Clarke L \& de Assis S 2016 Paternal overweight is associated with increased breast cancer risk in daughters in a mouse model. Scientific Reports 6 28602. (https://doi.org/10.1038/srep28602)

Genkinger JM, Kitahara CM, Bernstein L, Berrington de Gonzalez A, Brotzman M, Elena JW, Giles GG, Hartge P, Singh PN, StolzenbergSolomon RZ, et al. 2015 Central adiposity, obesity during early adulthood, and pancreatic cancer mortality in a pooled analysis of cohort studies. Annals of Oncology 26 2257-2266. (https://doi. org/10.1093/annonc/mdv355)
Hingorani SR, Petricoin EF, Maitra A, Rajapakse V, King C, Jacobetz MA, Ross S, Conrads TP, Veenstra TD, Hitt BA, et al. 2003 Preinvasive and invasive ductal pancreatic cancer and its early detection in the mouse. Cancer Cell 4 437-450. (https://doi.org/10.1016/S15356108(03)00309-X)

Huypens P, Sass S, Wu M, Dyckhoff D, Tschop M, Theis F, Marschall S, de Angelis MH \& Beckers J 2016 Epigenetic germline inheritance of diet-induced obesity and insulin resistance. Nature Genetics $\mathbf{4 8}$ 497-499. (https://doi.org/10.1038/ng.3527)

Incio J, Liu H, Suboj P, Chin SM, Chen IX, Pinter M, Ng MR, Nia HT, Grahovac J, Kao S, et al. 2016 Obesity-induced inflammation and desmoplasia promote pancreatic cancer progression and resistance to chemotherapy. Cancer Discovery 6 852-869. (https://doi. org/10.1158/2159-8290.CD-15-1177)

Jiao L, Berrington de Gonzalez A, Hartge P, Pfeiffer RM, Park Y, Freedman DM, Gail MH, Alavanja MC, Albanes D, Beane Freeman LE, et al. 2010 Body mass index, effect modifiers, and risk of pancreatic cancer: a pooled study of seven prospective cohorts. Cancer Causes and Control 21 1305-1314. (https://doi.org/10.1007/ s10552-010-9558-x)

Jimenez-Chillaron JC, Isganaitis E, Charalambous M, Gesta S, PentinatPelegrin T, Faucette RR, Otis JP, Chow A, Diaz R, Ferguson-Smith A, et al. 2009 Intergenerational transmission of glucose intolerance and obesity by in utero undernutrition in mice. Diabetes $\mathbf{5 8} 460-468$. (https://doi.org/10.2337/db08-0490)

Jirtle RL \& Skinner MK 2007 Environmental epigenomics and disease susceptibility. Nature Reviews Genetics 8 253-262. (https://doi. org/10.1038/nrg2045)

Kanda M, Sadakari Y, Borges M, Topazian M, Farrell J, Syngal S, Lee J, Kamel I, Lennon AM, Knight S, et al. 2013 Mutant TP53 in duodenal samples of pancreatic juice from patients with pancreatic cancer or high-grade dysplasia. Clinical Gastroenterology and Hepatology 11719. e5-730.e5. (https://doi.org/10.1016/j.cgh.2012.11.016)

Kopp JL, Von Figura G, Mayes E, Liu FF, Dubois CL, Morris JPT, Pan FC, Akiyama H, Wright CV, Jensen K, et al. 2012 Identification of Sox9-dependent acinar-to-ductal reprogramming as the principal mechanism for initiation of pancreatic ductal adenocarcinoma. Cancer Cell 22 737-750. (https://doi. org/10.1016/j.ccr.2012.10.025)

Li B \& Dewey CN 2011 RSEM: accurate transcript quantification from RNA-Seq data with or without a reference genome. $B M C$ Bioinformatics 12 323. (https://doi.org/10.1186/1471-2105-12-323)

Li D, Morris JS, Liu J, Hassan MM, Day RS, Bondy ML \& Abbruzzese JL 2009 Body mass index and risk, age of onset, and survival in patients with pancreatic cancer. JAMA 301 2553-2562. (https://doi. org/10.1001/jama.2009.886)

Linabery AM, Nahhas RW, Johnson W, Choh AC, Towne B, Odegaard AO, Czerwinski SA \& Demerath EW 2013 Stronger influence of maternal than paternal obesity on infant and early childhood body mass index: the Fels Longitudinal Study. Pediatric Obesity 8 159-169. (https://doi. org/10.1111/j.2047-6310.2012.00100.x)

Ling S, Chang X, Schultz L, Lee TK, Chaux A, Marchionni L, Netto GJ, Sidransky D \& Berman DM 2011 An EGFR-ERK-SOX9 signaling cascade links urothelial development and regeneration to cancer. Cancer Research 71 3812-3821. (https://doi.org/10.1158/0008-5472. CAN-10-3072)

Loche E, Blackmore HL, Carpenter AA, Beeson JH, Pinnock A, Ashmore TJ, Aiken CE, de Almeida-Faria J, Schoonejans JM, Giussani DA, et al. 2018 Maternal diet-induced obesity programmes cardiac dysfunction in male mice independently of post-weaning diet. Cardiovascular Research 114 1372-1384. (https://doi. org/10.1093/cvr/cvy082)

McPherson NO, Bell VG, Zander-Fox DL, Fullston T, Wu LL, Robker RL \& Lane M 2015 When two obese parents are worse than one! Impacts on embryo and fetal development. American Journal of (c) 2019 Society for Endocrinology Published by Bioscientifica Ltd. Printed in Great Britain 
Physiology: Endocrinology and Metabolism 309 E568-E581. (https://doi. org/10.1152/ajpendo.00230.2015)

Monteiro R \& Azevedo I 2010 Chronic inflammation in obesity and the metabolic syndrome. Mediators of Inflammation 2010289645. (https://doi.org/10.1155/2010/289645)

Ng SF, Lin RC, Laybutt DR, Barres R, Owens JA \& Morris MJ 2010 Chronic high-fat diet in fathers programs $\beta$-cell dysfunction in female rat offspring. Nature 467 963-966. (https://doi.org/10.1038/nature09491)

Nogueira L, Stolzenberg-Solomon R, Gamborg M, Sorensen TIA \& Baker JL 2017 Childhood body mass index and risk of adult pancreatic cancer. Current Developments in Nutrition 1 e001362. (https://doi.org/10.3945/cdn.117.001362)

Odermatt A 2011 The Western-style diet: a major risk factor for impaired kidney function and chronic kidney disease. American Journal of Physiology: Renal Physiology 301 F919-F931. (https://doi. org/10.1152/ajprenal.00068.2011)

Pandol S, Gukovskaya A, Edderkaoui M, Dawson D, Eibl G \& Lugea A 2012 Epidemiology, risk factors, and the promotion of pancreatic cancer: role of the stellate cell. Journal of Gastroenterology and Hepatology 27 (Supplement 2) 127-134. (https://doi. org/10.1111/j.1440-1746.2011.07013.x)

Risch HA 2019 Diabetes and pancreatic cancer: both cause and effect. Journal of the National Cancer Institute 111 1-2. (https://doi. org/10.1093/jnci/djy093)

Ritchie ME, Phipson B, Wu D, Hu Y, Law CW, Shi W \& Smyth GK 2015 Limma powers differential expression analyses for RNA-sequencing and microarray studies. Nucleic Acids Research 43 e47. (https://doi. org/10.1093/nar/gkv007)

Rodgers AB, Morgan CP, Leu NA \& Bale TL 2015 Transgenerational epigenetic programming via sperm microRNA recapitulates effects of paternal stress. PNAS 112 13699-13704. (https://doi.org/10.1073/ pnas.1508347112)
Rosenfeld CS 2015 Sex-specific placental responses in fetal development. Endocrinology 156 3422-3434. (https://doi. org/10.1210/en.2015-1227)

Santos S, Monnereau C, Felix JF, Duijts L, Gaillard R \& Jaddoe VWV 2018 Maternal body mass index, gestational weight gain, and childhood abdominal, pericardial, and liver fat assessed by magnetic resonance imaging. International Journal of Obesity $\mathbf{4 3}$ 581-593.

Sharma U, Conine CC, Shea JM, Boskovic A, Derr AG, Bing XY, Belleannee C, Kucukural A, Serra RW, Sun F, et al. 2016 Biogenesis and function of tRNA fragments during sperm maturation and fertilization in mammals. Science 351 391-396. (https://doi. org/10.1126/science.aad6780)

Shi M, He X, Wei W, Wang J, Zhang T \& Shen X 2015 Tenascin-C induces resistance to apoptosis in pancreatic cancer cell through activation of ERK/NF-kappaB pathway. Apoptosis 20 843-857. (https://doi.org/10.1007/s10495-015-1106-4)

van Kruijsdijk RC, van der Wall E \& Visseren FL 2009 Obesity and cancer: the role of dysfunctional adipose tissue. Cancer Epidemiology, Biomarkers and Prevention 18 2569-2578. (https://doi. org/10.1158/1055-9965.EPI-09-0372)

Watkins AJ, Sirovica S, Stokes B, Isaacs M, Addison O \& Martin RA 2017 Paternal low protein diet programs preimplantation embryo gene expression, fetal growth and skeletal development in mice. Biochimica et Biophysica Acta: Molecular Basis of Disease 1863 1371-1381. (https://doi.org/10.1016/j. bbadis.2017.02.009)

Whatcott CJ, Diep CH, Jiang P, Watanabe A, Lobello J, Sima C, Hostetter G, Shepard HM, Von Hoff DD \& Han H 2015 Desmoplasia in primary tumors and metastatic lesions of pancreatic cancer. Clinical Cancer Research 21 3561-3568. (https://doi. org/10.1158/1078-0432.CCR-14-1051)

Received in final form 6 March 2019

Accepted 13 March 2019

Accepted Preprint published online 13 March 2019 (c) 2019 Society for Endocrinology Published by Bioscientifica Ltd. Printed in Great Britain 